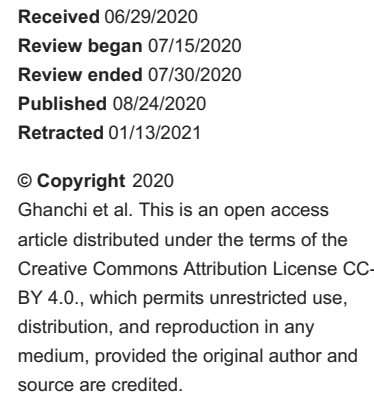

\section{Retracted: Effects of the COVID-19 Pandemic on Stroke Patients}

Hammad Ghanchi ${ }^{1}$, Ariel Takayanagi ${ }^{1}$, Paras Savla ${ }^{1}$, Omid R. Hariri ${ }^{2}$, Emilio C. Tayag ${ }^{3}$, Michael Schiraldi ${ }^{4,5}$, Lucille Jorgensen ${ }^{6}$, Dan E. Miulli ${ }^{7}$

1. Neurosurgery, Riverside University Health System Medical Center, Moreno Valley, USA 2. Neurosurgery, Kaiser Permanente-Orange County, Anaheim, USA 3. Neurology and Neurosurgery, Desert Regional Medical Center, Palm Springs, USA 4. Neurosurgery, Desert Regional Medical Center, Palm Springs, USA 5. Neurosurgery, Redlands Community Hospital, Redlands, USA 6. Stroke Program Coordinator, Arrowhead Regional Medical Center, Colton, USA 7. Neurosurgery, Arrowhead Regional Medical Center, Colton, USA

Corresponding author: Ariel Takayanagi, arieltaka@gmail.com

\section{This article has been retracted.}

Retraction date: January 13, 2021. Cite this retraction as Ghanchi H, Takayanagi A, Savla P, et al. (January 13, 2021) Retraction: Effects of the COVID-19 Pandemic on Stroke Patients. Cureus 13(1): r22. doi:10.7759/cureus.r22.

This article has been retracted as it includes data published without permission from the Get With the Guidelines database, which is property of American Heart Association (AHA). Although the authors' hospital had access to this database and they did receive IRB approval, the terms of use between the hospital and AHA do not allow publication of the data in a public sphere. The authors were hoping to receive retrospective approval from the AHA but have not been successful in getting this permission thus far. As a result this article has been retracted at the request of the AHA and with full agreement by the authors.

\section{Abstract}

\section{Introduction}

The severe acute respiratory syndrome coronavirus 2 (SARS2-CoV-2) induced pandemic (COVID-19 pandemic) has affected healthcare in all aspects, including stroke care. We sought to investigate this effect with analysis of our hospital's stroke treatment protocols as well as stroke volume on state, regional, and national levels.

\section{Methods}

This was a retrospective review of prospectively collected data from our stroke registry to assess the impact of the SARS2-CoV-2 induced pandemic on the volume of stroke patients presenting to our facility. Demographics collected included age, sex, race, National Institute of Health Stroke Scale (NIHSS) on admission, discharge modified Rankin Score (mRS), type of stroke (ischemic, hemorrhagic, or transient ischemic attack), time of symptom onset, and time to initial imaging. Data were also stratified by date and comparison was made between the intra-COVID-period (March and April 2020), pre-COVID period (March and April 2019), and peri-COVID period (January and February 2020). To determine stroke trends on a national level, we utilized the Get with the Guidelines (GWTG) stroke database to compare stroke volumes in the pre-COVID, peri-COVID, and intra-COVID periods between our hospital, all California hospitals, and the West and Pacific regions.

\section{Results}

There was a significant increase in last known well time (LKWT) to arrival to the emergency department (ED) (LKWT to door) as well as time from arrival to the ED to obtaining a computed tomography (CT) of the head (door to CT) in March 2020 compared to 2019 ( $\mathrm{p}=0.0220$ and $\mathrm{p}=0.0475$, respectively). There were significantly fewer transient ischemic attacks (TIAs) in California hospitals as well as in March and April 2020 in comparison to January and February 2020 ( $p=0.0417)$. Similarly, there were significantly fewer TIAs in March and April 2019 compared to March and April 2020 ( $\mathrm{p}=0.0360)$. The decrease in TIAs was also seen at our hospital in both time frame comparisons as well as in West Regional Hospitals in March and April 2020 compared to March and April 2019 ( $\mathrm{p}=0.0111, \mathrm{p}=0.0215$, and $\mathrm{p}=0.0414$, respectively).

\section{Conclusion}

Stroke care has been disrupted by the COVID-19 pandemic worldwide. We identified a delay in LKWT to door as well as time from door to CT in March 2020 compared to March 2019 at our institution. There was a statistically significant decrease in final diagnosis of TIA at our hospital, all California hospitals, and all West Regional hospitals during the March-April 2020 window, suggesting that some patients with minor stroke symptoms may not be presenting to the hospital in the midst of the pandemic. Strategies to minimize 
Categories: Neurology, Infectious Disease, Neurosurgery

Keywords: stroke, diagnostic delay, nihss, covid-19, tpa, coronavirus quarantine, transient ischemic attack, patient delay, stroke guidelines, stroke protocol

\section{Introduction}

The coronavirus disease 2019 (COVID-19) pandemic caused by the severe acute respiratory syndrome coronavirus 2 (SARS2-CoV-2) has had detrimental effects on not only our economy, but it has also affected treatment of other serious diseases. Recent studies have been published demonstrating the adverse effects this pandemic has had thus far on the treatment of stroke patients [1-4].

Over the past few decades, great measures have been taken to decrease time from stroke symptom onset to treatment in order to maximize recovery [5]. However, during the COVID-19 pandemic, the public has been discouraged from leaving their homes to minimize risk of infection and slow the spread of the disease. A state of emergency was issued by many state governments early March 2020 which evolved into stay-athome orders. The orders were first placed in California mid-March 2020 and slowly spread to the other states in the following weeks [6]. At the same time, hospitals across the nation started to prepare for a surge in hospitalizations and began focusing efforts on providing COVID-directed care. Since the pandemic began, delays in presentation and decreases in patients receiving tissue plasminogen activator (tPA) have been reported [2]. We performed a retrospective study using our institutional stroke registry to investigate preand intra-hospital delays during the COVID period. We then compared our institutional data to the Get with the Guidelines (GWTG) database to analyze our experience by comparing regional and national trends in stroke volume $[7,8]$.

\section{Materials And Methods}

This was a retrospective review of the stroke registry at our institution, a Level 1 primary stroke center as certified by the Joint Commission/American Stroke Association in Colton, California, to assess what impact the SARS2-Cov-2 outbreak had on the number of patients presenting with stroke to our hospital. All patients with a final diagnosis of ischemic stroke, transient ischemic attack (TIA), subarachnoid hemorrhage (SAH), or intraparenchymal hemorrhage (IPH) were reviewed.

Demographics were collected including age, sex, race, National Institute of Health Stroke Scale (NIHSS) on admission, discharge modified Rankin Score (mRS), type of stroke (ischemic, hemorrhagic, or transient ischemic attack), last known well time (LKWT), and time to initial cranial imaging. Data was also stratified by date into three groups: (a) intra-COVID period (March and April 2020), (b) pre-COVID period (March and April 2019), and peri-COVID period (January and February 2020).

To determine stroke trends on a regional and national level, we utilized the GWTG database to compare stroke volumes in the same three time periods between our hospital, all California hospitals, all Pacific hospitals (Alaska, Washington, Oregon, California, and Hawaii), all Western hospitals (Pacific plus Montana, Idaho, Wyoming, Nevada, Utah, Colorado, Arizona, and New Mexico), and all hospitals in the United States that submit data to the registry. The numbers of patients presenting to these hospitals with a final diagnosis of ischemic stroke, TIA, SAH, or IPH were reviewed.

The primary endpoint of this study is to compare whether there was a significant change in the number of patients presenting to our institution with stroke during the SARS2-CoV-2 pandemic. The secondary endpoints were to assess differences in severity of stroke, delays in imaging, delays in presentation, and changes in stroke incidence.

Statistical analysis was performed using Student's t-Test to compare the sample means and assess for any significant differences in the patient volumes, times for treatment, and severity of strokes.

\section{Results}

\section{County Hospital Stroke Results}

On our review, 348 patients presented to our institution between during the first four months in 2019 and 302 patients in 2020 with symptoms concerning for stroke resulting in activation of our stroke protocols. Of these patients, a total of 262 were found to have a final diagnosis of stroke (ischemic, hemorrhagic, or TIA) on discharge; 135 patients in 2019 and 127 patients in 2020. The mean age of both groups was 60 years with 2019 ranging from 20 to 99 years old and 2020 ranging from 21 to 93 years old. For gender, no significant differences were seen, with 2019 having 67 (49.6\%) males and 68 (50.4\%) females and 2020 having 57 (44.9\%) males and 70 (55.1\%) females. 


\section{Cureus}

There was a significant increase in last known well time (LKWT) to arrival to the emergency department (ED) (LKWT to door) as well as time from arrival to the ED to obtaining a computed tomography (CT) of the head (door to CT) in March 2020 compared to 2019 ( $\mathrm{p}=0.0220$ and $\mathrm{p}=0.0475$, respectively) (Table 1, Figure 1, Figure 2). There was no difference in LKWT to door or door to CT between April 2019 and 2020. Additionally, while the NIH score was increased in April 2020 compared to 2019 from 4.14 to 8.16, the difference was not statistically significant $(\mathrm{p}=0.17)$ (Table 1, Figure 3).

\begin{tabular}{|c|c|c|c|c|c|c|}
\hline \multirow[t]{2}{*}{ Month } & Mar '19 & Mar '20 & & Apr'19 & Apr '20 & \\
\hline & Mean & Mean & $\mathrm{p}$-value & Mean & Mean & $p=$ value \\
\hline \multicolumn{7}{|l|}{ Timing } \\
\hline LKWT to door (min) & 836 & 2561 & 0.0220 & 1457 & 1039 & 0.4623 \\
\hline Door to CT (min) & 17 & 76 & 0.0475 & 50 & 57 & 0.8321 \\
\hline \multicolumn{7}{|c|}{ Admission Stroke Severity and Discharge Functional Status } \\
\hline $\mathrm{NIH}$ & 6.27 & 5.74 & 0.8173 & 4.14 & 8.16 & 0.1767 \\
\hline mRS & 2.08 & 2.43 & 0.4900 & 2.32 & 2.32 & 0.9882 \\
\hline
\end{tabular}

\section{TABLE 1: Comparison of pre-COVID versus COVID period timing and stroke severity}

Abbreviations: LKWT: last known well time, NIH: National Institutes of Health, H\&H: Hunt and Hess score, ICH: Intraparenchymal hemorrhage score, mRS: modified Rankin scale, Pre-COVID: March, April 2019, COVID period: March, April 2020, N/A: not applicable

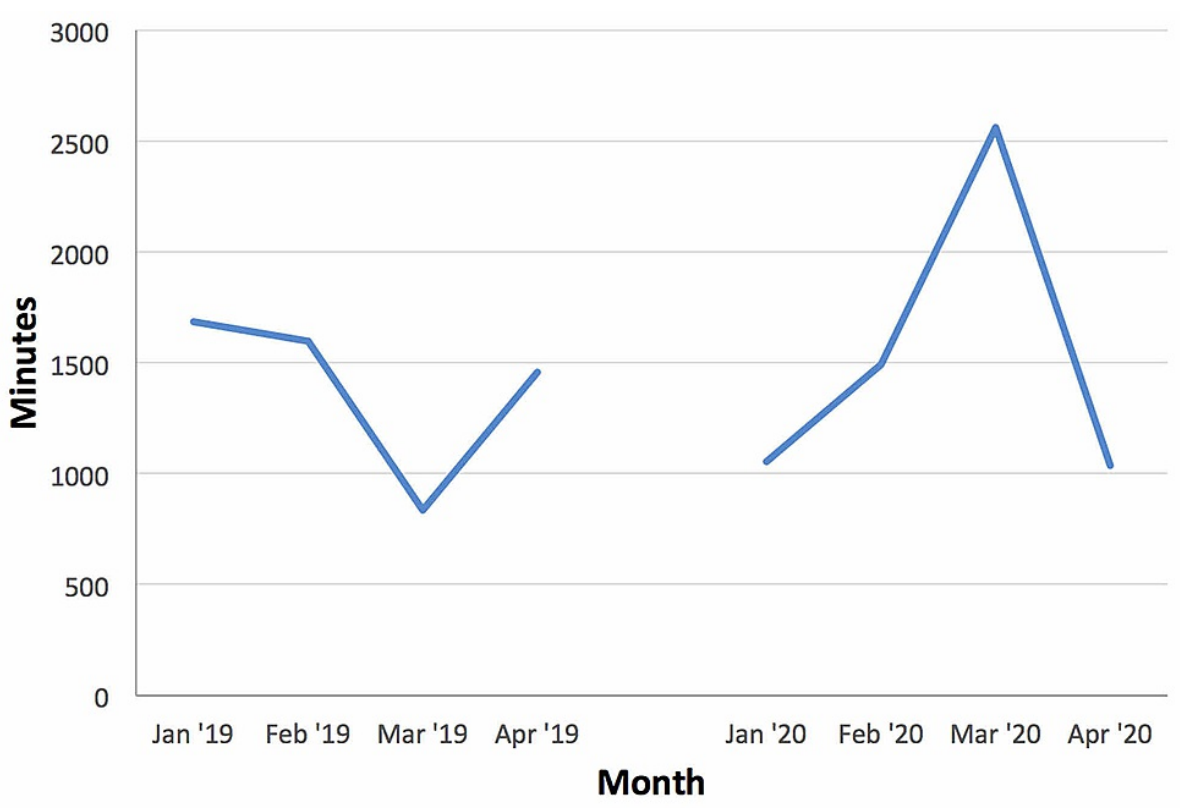

FIGURE 1: Last Known Well Time to Door 


\section{Cureus}

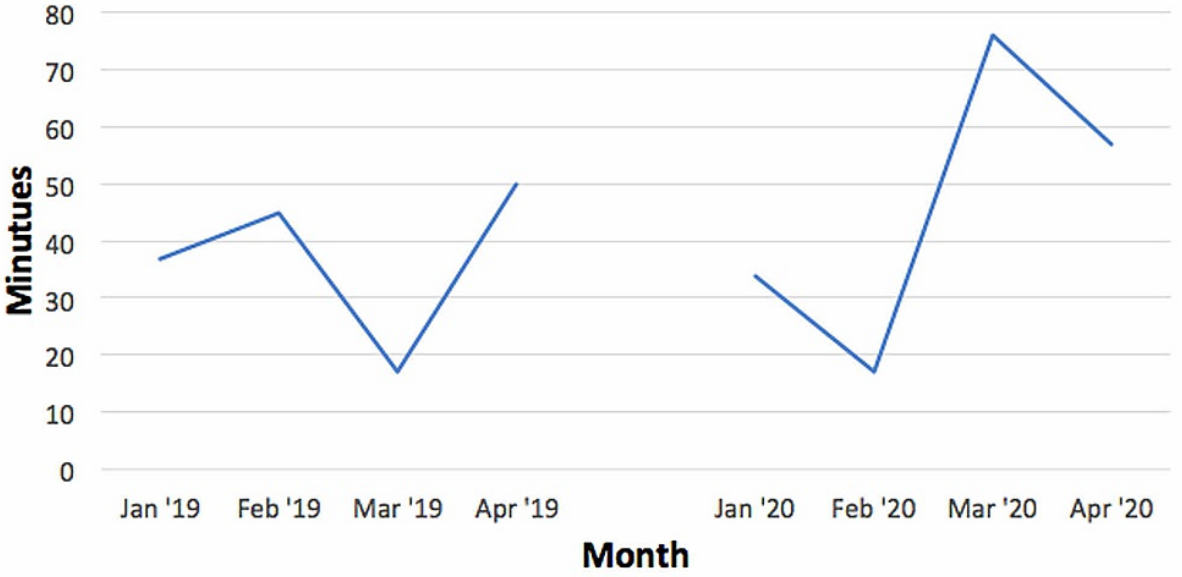

\section{FIGURE 2: Time from Door to CT}

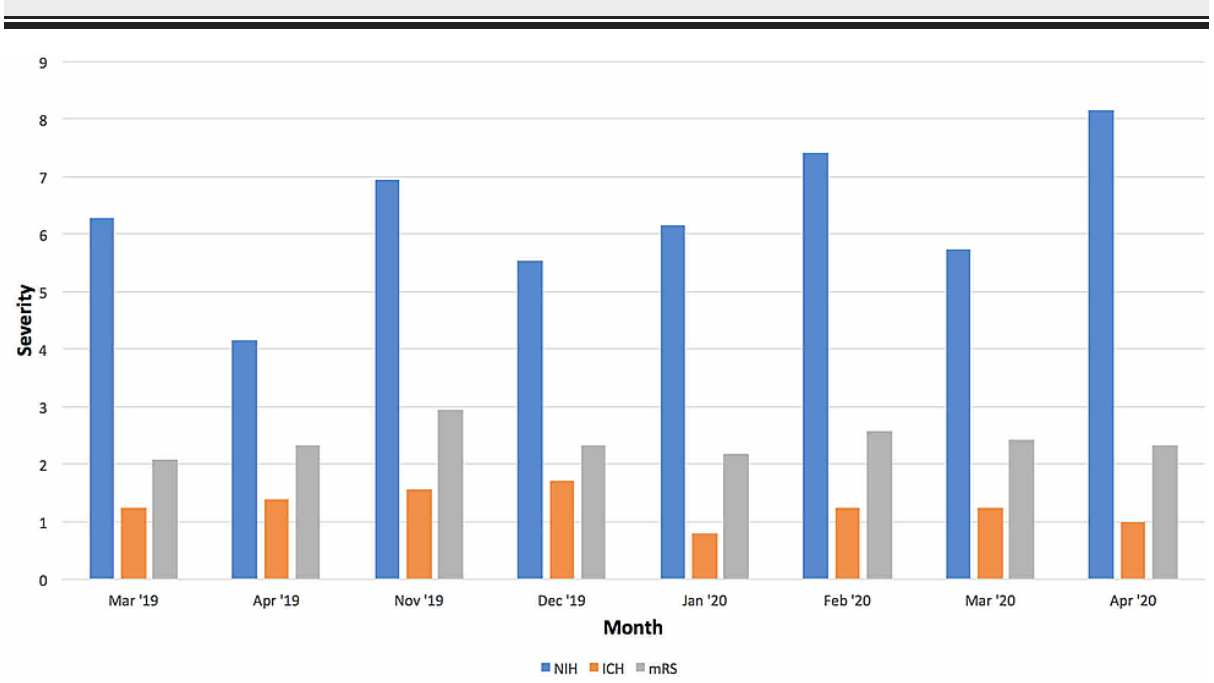

FIGURE 3: Stroke Severity and Functional Status

\section{National, Regional, and Local Stroke Volume}

Stroke volumes in January through April of 2019 and 2020 for our hospital as well as regional and national data are listed in Table 2. Comparisons of stroke volumes in the peri-COVID period and the COVID period as well as pre-COVID period and COVID-period are demonstrated in Table 3. There were significantly fewer TIAs in California hospitals as well as in March and April 2020 in comparison to January and February 2020 $(\mathrm{p}=0.0417)$. Similarly, there were significantly fewer TIAs in March and April 2019 compared to March and April 2020 ( $p=0.0360)$. The decrease in TIAs was also seen at our hospital in both time frame comparisons $(\mathrm{p}=0.0111, \mathrm{p}=0.0215)$ as well as in West Regional Hospitals when March and April 2020 was compared to March and April 2019 ( $\mathrm{p}=0.0414)$. There was also a significant decrease in ischemic strokes in the Pacific region from March/April 2019 to March/April 2020 and from January/February 2020 to March/April 2020 ( $\mathrm{p}=0.0462$ and $\mathrm{p}=0.0383$, respectively). 


\section{Cureus}

\begin{tabular}{|c|c|c|c|c|c|c|c|c|}
\hline \multirow{2}{*}{ Region } & \multicolumn{2}{|l|}{ IS } & \multicolumn{2}{|l|}{ TIA } & \multicolumn{2}{|l|}{ SAH } & \multicolumn{2}{|l|}{$\mathrm{ICH}$} \\
\hline & 2019 & 2020 & 2019 & 2020 & 2019 & 2020 & 2019 & 2020 \\
\hline \multicolumn{9}{|l|}{ ARMC } \\
\hline January & 14 & 14 & 13 & 15 & 3 & 0 & 3 & 9 \\
\hline February & 21 & 15 & 8 & 16 & 1 & 2 & 7 & 10 \\
\hline March & 11 & 11 & 12 & 6 & 1 & 4 & 11 & 9 \\
\hline April & 12 & 14 & 13 & 4 & 0 & 1 & 5 & 5 \\
\hline \multicolumn{9}{|c|}{ CA Hospitals } \\
\hline January & 4086 & 4325 & 658 & 700 & 277 & 270 & 909 & 912 \\
\hline February & 3705 & 3881 & 641 & 659 & 271 & 238 & 868 & 785 \\
\hline March & 4115 & 3475 & 717 & 458 & 275 & 202 & 866 & 747 \\
\hline April & 3987 & 2284 & 680 & 349 & 257 & 160 & 825 & 508 \\
\hline \multicolumn{9}{|c|}{ Pacific Region } \\
\hline January & 5821 & 6037 & 945 & 940 & 387 & 360 & 1233 & 1211 \\
\hline February & 5176 & 5408 & 929 & 894 & 363 & 304 & 1164 & 1083 \\
\hline March & 5831 & 4941 & 1036 & 600 & 366 & 284 & 1135 & 997 \\
\hline April & 5636 & 3263 & 951 & 426 & 361 & 218 & 1087 & 716 \\
\hline \multicolumn{9}{|c|}{ West Regional } \\
\hline January & 7901 & 8172 & 1249 & 1189 & 540 & 492 & 1611 & 1623 \\
\hline February & 6982 & 7372 & 1194 & 1126 & 494 & 422 & 1511 & 1447 \\
\hline March & 7824 & 6634 & 1344 & 764 & 496 & 380 & 1505 & 1329 \\
\hline April & 7599 & 4474 & 1222 & 525 & 501 & 280 & 1459 & 951 \\
\hline \multicolumn{9}{|c|}{ All Hospitals } \\
\hline January & 40644 & 42660 & 6749 & 7150 & 2383 & 2353 & 7305 & 7287 \\
\hline February & 36946 & 37999 & 6194 & 6556 & 2135 & 1940 & 6489 & 6233 \\
\hline March & 40403 & 34986 & 7340 & 4940 & 2269 & 1960 & 6890 & 5819 \\
\hline April & 40099 & 22748 & 6877 & 2856 & 2174 & 1431 & 6538 & 4307 \\
\hline
\end{tabular}

\section{TABLE 2: National, regional, and local stroke volumes in 2019 and 2020}

Abbreviations: ARMC: Arrowhead Regional Medical Center; IS: ischemic stroke; TIA: transient ischemic attack <24 hours; SAH: subarachnoid hemorrhage; ICH: intraparenchymal hemorrhage; All hospitals: all hospitals in the US; Pacific: Alaska, Washington, Oregon, California, Hawaii; West Region: Pacific region plus Montana, Idaho, Wyoming, Nevada, Utah, Colorado, Arizona, New Mexico 


\section{Cureus}

\begin{tabular}{|c|c|c|c|c|}
\hline & IS & TIA & SAH & $\mathrm{ICH}$ \\
\hline \multicolumn{5}{|l|}{ ARMC } \\
\hline Jan Feb '20 vs Mar Apr '20 & 0.3333 & 0.0111 & 0.4929 & 0.3491 \\
\hline Mar Apr '19 vs Mar Apr '20 & 0.5918 & 0.0215 & 0.3333 & 0.8075 \\
\hline \multicolumn{5}{|l|}{ California Hospitals } \\
\hline Jan Feb '20 vs Mar Apr '20 & 0.1941 & 0.0417 & 0.1097 & 0.2440 \\
\hline Mar Apr ' 19 vs Mar Apr '20 & 0.1896 & 0.0360 & 0.0653 & 0.2140 \\
\hline \multicolumn{5}{|l|}{ Pacific Region } \\
\hline Jan Feb '20 vs Mar Apr '20 & 0.0462 & 0.2022 & 0.1729 & 0.1986 \\
\hline Mar Apr '19 vs Mar Apr '20 & 0.0383 & 0.0767 & 0.0781 & 0.2143 \\
\hline \multicolumn{5}{|l|}{ West Regional } \\
\hline Jan Feb '20 vs Mar Apr '20 & 0.1940 & 0.0534 & 0.1729 & 0.1986 \\
\hline Mar Apr '19 vs Mar Apr '20 & 0.1853 & 0.0414 & 0.0781 & 0.2143 \\
\hline \multicolumn{5}{|l|}{ All Hospitals } \\
\hline Jan Feb '20 vs Mar Apr '20 & 0.2221 & 0.1123 & 0.3111 & 0.2069 \\
\hline Mar Apr '19 vs Mar Apr '20 & 0.2040 & 0.0950 & 0.1894 & 0.1673 \\
\hline
\end{tabular}

TABLE 3: Comparision of national, regional, and local stroke volumes in pre-COVID, peri-COVID, and COVID periods ( $p$ values)

Abbreviations: ARMC: Arrowhead Regional Medical Center; IS: ischemic stroke; TIA: transient ischemic attack <24 hours; SAH: subarachnoid hemorrhage; ICH: intraparenchymal hemorrhage; All hospitals: all hospitals in the US; Pacific: Alaska, Washington, Oregon, California, Hawaii; West Region: Pacific region plus Montana, Idaho, Wyoming, Nevada, Utah, Colorado, Arizona, New Mexico

\section{Discussion}

SARS2-CoV-2 is the first virus in modern history to cause a global economic shutdown. In addition to its effect on the economy, SARS2-CoV-2 also caused major disruptions in healthcare as many hospitals were forced to cancel elective surgeries and postpone outpatient and specialty appointments to conserve personal protective equipment (PPE) and reduce exposure risk. Teo et al. has suggested that the recent change in healthcare has had a significant impact on care of stroke patients [2].

A delay in management of stroke patients may be related to the COVID-19 pandemic. Schirmer et al. compared stroke severity and time to presentation between patients who presented with stroke in the preCOVID and intra-COVID period at twelve institutions, and the corresponding months from one year prior [9]. There was a significant increase in time interval between symptom onset and time to presentation to a stroke center. Patients in the baseline period presented in 442 minutes (mins), while intra-COVID period patients presented in 603 mins. In the present study, the LKWT to ED door significantly increased in March 2020 compared to March 2019 ( 2561 mins versus 836 mins, $p=0.0220$ ); this corresponds to when the State of Emergency was declared and most states issued stay-at-home orders.

Another study showed similar results with a significant increase in LKWT to door time during the COVID-19 period, and more importantly a significant reduction in patients who arrived within the 4.5 hour tPA window [2]. Similarly, Kerleroux et al. also found that patients who were treated during the same time period were less likely to receive tPA [3]. While the cause of delay in presentation is unclear, it may be related to reluctance to go to the hospital due to fear of infection [2]. Another possibility is that there is a delay in ambulance response times, but further studies will be necessary to identify the cause of the delays. 
Mechanical thrombectomy times have also been adversely affected. Kerleroux et al. showed a significant decrease in thrombectomy volumes as well as a significant increase in time between imaging and groin puncture <0.001) [3]. The authors hypothesize that the decrease in number of thrombectomies may be related to the saturation of transport systems, which may prevent patients from being transferred to facilities capable of stroke thrombectomies in a timely manner [3]. Institutional delays during the pandemic, as seen in our study, may result in further delays.

We also found a statistically significant increase in time from door to CT when March 2019 and March 2020 were compared ( 17 mins to 76 mins, $\mathrm{p}=0.0475$ ). When patients who are suspected of having SARS2-CoV-2 infection undergo a CT study at our hospital, the CT scanner room must undergo strict disinfecting protocols before another patient can be scanned. This may have caused delays in CT scans overall. Interestingly, there was no significant difference in time from door to CT between April 2019 and April 2020 $(\mathrm{p}=0.8321)$. This corresponds with the development and distribution of the rapid COVID-19 test during April which may have helped to minimize CT downtime. Furthermore, our times for April 2019 were higher than average, which may account for the decrease being smaller than the decrease from March 2019 to March 2020. Turin et al. have also shown in the past that incidence of stroke in the spring season is higher [10]. Another delay that may be related to the pandemic is the additional time needed for donning and doffing of personal protective gear. However, these explanations are anecdotal and future studies are necessary to elucidate the causes of in-hospital delays.

There was no significant difference in LKWT to door time when April of 2020 was compared to April 2019 $(\mathrm{p}=0.4623)$. However, we do see a decrease in number of patients diagnosed with stroke during this month, trending down from March in almost all regions (Table 2). Moreover, there was a statistically significant decrease in final diagnosis of TIA at our hospital, all California hospitals, and all West Regional hospitals during the March-April 2020 window (Table 3). Those with minor or transient stroke-like symptoms (i.e. TIAs) may have avoided presenting to the hospital due to fear of infection. With regards to severity of stroke, we found that NIHSS scores were higher in April 2020 (8.16) than April 2019 (4.14), but the difference did not reach statistical significance $(\mathrm{p}=0.1767)$. There was also no significant difference in NIHSS score between March 2019 and March 2020 ( $\mathrm{p}=0.8173$ ). This was consistent with Schirmer et al. who reported no change in NIH score from 2019 [9]. Finally, the patient outcomes as measured by mRS were similar between March and April 2019 and March and April of 2020 (p=0.49) (Table 1).

The AHA/ASA Stroke Council Leadership has published guidelines for care of stroke patients during the COVID-19 pandemic. While the recommendation is to continue to follow stroke protocols and aim to minimize time to treatment, they suggest that the guidelines should be treated as goals rather than expectations given limited resources and staffing [11]. Other efforts have been made to streamline stroke care during the pandemic. Khosravani et al. published a "Protected Code Stroke” algorithm for managing patients with suspected COVID-19 infection [12]. The authors recommend infectious screening in the field prior to arrival, appropriate use of personal protective equipment, and strategies to minimize exposure during airway management such as early intubation [12]. The Neuroscience in Anesthesiology and Critical Care and Society of Neurointerventional Surgery have recommended rapid COVID-19 testing prior to most neurointerventional procedures. However, given that stroke thrombectomies must be done emergently due to the time-sensitive nature of cerebral ischemia, patients should be assumed to be COVID-19 positive and only essential personnel should be present in the angiography suite [13]. Others have recommended consolidating imaging studies to minimize staff exposure [14].

\section{Conclusions}

Stroke care has been disrupted by the COVID-19 pandemic worldwide. We identified a delay in LKWT to door as well as time from door to CT in March 2020 compared to March 2019 at our institution. There was a statistically significant decrease in final diagnosis of TIA at our hospital, all California hospitals, and all West Regional hospitals during the March-April 2020 window, suggesting that some patients with minor stroke symptoms may not be presenting to the hospital in the midst of the pandemic. Strategies to minimize delays in care and maximize functional recovery must continue to evolve as new challenges are met during the COVID-19 pandemic.

\section{Additional Information \\ Disclosures}

Human subjects: All authors have confirmed that this study did not involve human participants or tissue. Animal subjects: All authors have confirmed that this study did not involve animal subjects or tissue. Conflicts of interest: In compliance with the ICMJE uniform disclosure form, all authors declare the following: Payment/services info: All authors have declared that no financial support was received from any organization for the submitted work. Financial relationships: All authors have declared that they have no financial relationships at present or within the previous three years with any organizations that might have an interest in the submitted work. Other relationships: All authors have declared that there are no other relationships or activities that could appear to have influenced the submitted work. 


\section{References}

1. Yaghi S, Ishida K, Torres J, et al.: SARS2-CoV-2 and stroke in a New York healthcare system . Stroke. 2020, 51:2002-2011. 10.1161/STROKEAHA.120.030335

2. Teo K-C, Leung WC, Wong Y-K, Liu RK, et al.: Delays in stroke onset to hospital arrival time during COVID19. Stroke. 2020, 7:2228-2231. 10.1161/STROKEAHA.120.030105

3. Kerleroux B, Fabacher T, Bricout N, et al.: Mechanical thrombectomy for acute ischemic stroke amid the COVID-19 outbreak. Stroke. 2020, 51:2012-7. 10.1161/STROKEAHA.120.030373

4. Zhao J, Li H, Kung D, Fisher M, Shen Y, Liu R: Impact of the COVID-19 epidemic on stroke care and potential solutions. Stroke. 2020, 51:1996-2001. 10.1161/STROKEAHA.120.030225

5. Meretoja A, Strbian D, Mustanoja S, Tatlisumak T, Lindsberg PJ, Kaste M: Reducing in-hospital delay to 20 minutes in stroke thrombolysis. Neurology. 2012, 79:306-13. 10.1212/WNL.0b013e31825d6011

6. See Which States and Cities Have Told Residents to Stay at Home - The New York Times . (2020). Accessed: Jun 22 2020: https://www.nytimes.com/interactive/2020/us/coronavirus-stay-at-home-order.html.

7. Hoyer C, Ebert A, Huttner HB, et al.: Acute stroke in times of the COVID-19 pandemic: a multicenter study . Stroke. 2020, 51:2224-7. 10.1007/s00406-020-01151

8. Meza HT, Lambea Gil Á, Saldaña AS, et al.: Impact of COVID-19 outbreak on ischemic stroke admissions and in-hospital mortality in North-West Spain. Int J Stroke. 2020, 10.1177/1747493020938301

9. Schirmer CM, Ringer AJ, Arthur AS, et al.: Delayed presentation of acute ischemic strokes during the COVID-19 crisis. J Neurointerv Surg. 2020, 12:639-642. 10.1136/neurintsurg-2020-016299

10. Turin TC, Kita Y, Murakami Y, et al.: Higher stroke incidence in the spring season regardless of conventional risk factors: Takashima Stroke Registry, Japan, 1988-2001. Stroke. 2008, 39:745-52. 10.1161/STROKEAHA.107.495929

11. The AHA/ASA Stroke Council Leadership: Temporary emergency guidance to US stroke centers during the coronavirus disease 2019 (COVID-19) pandemic. Stroke. 2020, 51:1910-1912. doi/10.1161/STROKEAHA.120.030023

12. Khosravani H, Rajendram P, Notario L, et al.: Protected code stroke. Stroke. 2020, 51:1891-5. 10.1161/STROKEAHA.120.029838

13. Flexman AM, Abcejo AS, Avitsian R, et al.: Neuroanesthesia practice during the COVID-19 pandemic: recommendations from Society for Neuroscience in Anesthesiology and Critical Care (SNACC). J Neurosurg Anesthesiol. 2020, 32:

14. Dafer RM, Osteraas ND, Biller J: Acute stroke care in the coronavirus disease 2019 pandemic . J Stroke Cerebrovasc Dis. 2020, 29:104881. 10.1016/j.jstrokecerebrovasdis.2020.104881 